\title{
Towards Bose-Einstein Condensation of Semiconductor Excitons: The Biexciton Polarization Effect
}

\author{
D. Hägele, ${ }^{*}$ S. Pfalz, and M. Oestreich \\ Institut für Festkörperphysik, Leibniz Universität Hannover, Appelstraße 2, D-30167 Hannover, Germany
} (Received 30 April 2009; revised manuscript received 14 August 2009; published 30 September 2009)

\begin{abstract}
We theoretically predict a strong influence of stimulated exciton-exciton scattering on semiconductor luminescence. The stimulated scattering causes circularly polarized instead of unpolarized emission at the biexciton emission line in a degenerate gas of partly spin polarized excitons. The biexciton polarization effect increases with increasing exciton densities and decreasing temperatures and approaches almost unity in the ultimate case of Bose-Einstein condensation. Time- and polarization-resolved luminescence measurements evidence the biexciton polarization effect both in $\mathrm{ZnSe}$ and GaAs quantum wells.
\end{abstract}

DOI: 10.1103/PhysRevLett.103.146402

PACS numbers: 71.35.Lk, 72.25.Fe, 78.67.De

Bose-Einstein condensation (BEC) in atoms is now routinely validated by a set of measurable effects like the velocity distribution of the atoms [1] or more advanced coherence effects [2]. Proving BEC of bound electron hole pairs - so-called excitons - in semiconductors is in a way more challenging, although the BEC transition temperatures for excitons can be many orders of magnitude higher than in atoms. In 1962 Blatt, Böer, and Brandt predicted that optically created excitons in semiconductors undergo Bose-Einstein condensation at sufficiently high densities and low temperatures [3]. The critical temperature for condensation, $T_{c} \propto n^{2 / 3} m^{-1}$, depends on particle density $n$ and the effective exciton mass $m$. Compared to atoms, $T_{c}$ in semiconductors is about 8 orders of magnitude higher, where the lower effective exciton mass contributes about 5 orders and the higher achievable exciton densities about 3 orders. The resulting $T_{c}$ 's in common semiconductors can be as high as a few kelvins. Blatt, Böer, and Brandt suggested already in their pioneering work a narrowing of the homogeneous photoluminescence (PL) linewidth as a signature of BEC that is caused by the absence of exciton scattering within the condensate. This criterion, however, cannot be applied to most semiconductors, especially the prominent candidates $\mathrm{Cu}_{2} \mathrm{O}$ and $\mathrm{CuCl}$, as their inhomogeneous linewidths, which are caused by strain, are generally larger than the homogeneous linewidth [4]. Alternatively, Fernández-Rossier, Tejedor, and Merlin suggested measuring the photon statistics of PL from a condensate which should exhibit nonthermal laserlike behavior [5]. Detection of such signatures, however, does require sophisticated quantum optical methods. A number of interesting effects towards BEC have been found in coupled quantum wells [6]. However, their interpretation is "notoriously complex," as stated in Ref. [7]. BEC of excitons has to be contrasted with BEC of polaritons in semiconductor microcavities which has recently grown into a mature field [8]. Unlike in the present Letter, the strong exciton light coupling is a vital prerequisite for condensation which results in the exciting phenomenon of spatial coherence and non- linear enhancement of circular polarized light emission even far below the laser threshold $[9,10]$.

In the following we numerically find a novel effect in the luminescence of a gas of partly spin polarized semiconductor excitons. The degree of circular polarization of light emitted from bound pairs of excitons with opposite spins (so-called biexcitons [11]) turns from zero to finite at low temperatures and high exciton densities. This biexciton polarization effect (BPE) challenges intuition as biexcitons possess zero total spin. We analytically show that the BPE has its origin in stimulated scattering of excitons reaching its maximum in the case of BEC. First experiments in $\mathrm{ZnSe}$ and GaAs quantum wells (QWs) exhibit an onset of a $5 \%$ $\mathrm{BPE}$ at a sample temperature of $10 \mathrm{~K}$ and elevated exciton densities in accordance with theory. The following treatment of semiconductor luminescence complements existing theories [12,13], as it is based on simple assumptions, includes multi-exciton effects, accounts for the exciton spin structure, and allows for the calculation of PL spectra at finite temperatures. Choosing the QW growth direction $z$ as the quantization direction, conduction band electrons with spin $+1 / 2$ and $-1 / 2$ and heavy holes in the valence band with spin $+3 / 2$ and $-3 / 2$ form exciton states with total spin $+2,+1,-1$, and -2 . We regard only optically active excitons with spin \pm 1 in the $1 s$ state in the model and neglect the so-called dark excitons with spin \pm 2 and higher excited states. A justification will be given below. The one-dimensional model consists of $N$ adjacent sites in configuration space with a distance of one exciton diameter. The annihilation operators are given by $X_{j, s}$ for an exciton at site $j$ with spin $s= \pm 1$ in the site representation and by $x_{k, s}=N^{-1 / 2} \sum_{j} X_{j, s} \exp [2 \pi i(k j / N)]$ in the momentum representation. The model Hamiltonian

$$
H=\sum_{k, s= \pm 1} E_{k} x_{k, s}^{\dagger} x_{k, s}+\sum_{j} V_{B} X_{j,+1}^{\dagger} X_{j,+1} X_{j,-1}^{\dagger} X_{j,-1}
$$

includes the dispersion relation $E_{k}$ for excitons and an onsite binding potential $V_{B}<0$ for biexciton formation (for a very similar Hamiltonian see Ref. [14]). The operator 
commutation relations were chosen to exhibit both bosonic and fermionic properties $X_{j, s} X_{l, s}=X_{l, s} X_{j, s}$ and $X_{j, s}^{2}=0$, respectively. The second relation regards the fact that due to Pauli blocking no more than one exciton with spin $s$ may occupy a certain site (hard-core bosons). The on-site potential reflects the fact that formation of biexcitons is (like the formation of the $\mathrm{H}_{2}$ molecule) governed by short range exchange interaction. A coexistence of bright and dark excitons in close spatial vicinity is energetically not favorable because Pauli exclusion-mediated by either electrons or holes with equal spins-leads to lateral confinement which increases kinetic energy. The $2 s, 2 p, \ldots$ states of excitons are also energetically unfavorable. The above model with $1 s$ excitons of spin $s= \pm 1$ captures therefore the physics of the regions with optically active excitons at moderate to low temperatures.

Photoluminescence spectra are calculated numerically using the above Hamiltonian for $N=7$, where $k=$ $-3,-2, \ldots, 3, \quad E_{k} / \mathrm{eV}=2.8+0.011\{k /[(N+1) / 2]\}^{2}$, and $V_{B}=-8 \mathrm{meV}$ mimic the parameters for a $\mathrm{ZnSe}$ quantum well [15]. The polarization dependent PL spectra are obtained from

$$
I_{s}(\epsilon)=\sum_{i, f}\left\langle\psi_{i}|\rho| \psi_{i}\right\rangle\left|\left\langle\psi_{f}\left|D_{s}\right| \psi_{i}\right\rangle\right|^{2} \lambda\left[\epsilon-\left(\epsilon_{i}-\epsilon_{f}\right)\right],
$$

where $\rho \propto \exp \left[-\left(H-\mu_{1} n_{1}-\mu_{-1} n_{-1}\right) /\left(k_{B} T\right)\right]$ is the density matrix, $\mu_{1}$ and $\mu_{-1}$ are the chemical potentials for spin \pm 1 excitons, the operator $n_{s}=\sum_{j} X_{j, s}^{\dagger} X_{j, s}$ counts the number of excitons with spin $s$, and $D_{s}=d \sum_{j} X_{j, s}$ is the dipole operator in rotating wave approximation that couples to light with angular momentum $s= \pm 1$ (circular polarization). The double sum runs over all $2^{14}$ multiexciton eigenstates $\left|\psi_{i}\right\rangle$ and $\left|\psi_{f}\right\rangle$ which were obtained from exact numerical diagonalization of $H$. The energy difference $\epsilon_{i}-\epsilon_{f}$ of the initial and final state determines the spectral position of the emission line $\lambda$, where a linewidth of $0.33 \mathrm{meV}$ was chosen. The implicit assumption of thermal equilibrium of $\rho$ is justified in GaAs based quantum wells for densities of about $10^{10} \mathrm{~cm}^{-2}$ or higher [16] and in ZnSe based quantum wells for about $10^{11} \mathrm{~cm}^{-2}$ due to rapid exciton-exciton scattering [17].

Figures 1(a)-1(d) show calculated spectra for varying temperatures, different densities, and a fixed degree of exciton polarization $\left(\left\langle n_{1}\right\rangle-\left\langle n_{-1}\right\rangle\right) /\left(\left\langle n_{1}\right\rangle+\left\langle n_{-1}\right\rangle\right)=$ 0.25 . Figure 1(top) depicts the degree of biexciton polarization for temperatures between 3 and $50 \mathrm{~K}$ and densities between 0 and 1.6 excitons per site. We indicated the corresponding positions of spectra (a), (b), and (c) in this diagram. All spectra exhibit two distinct features corresponding to the exciton line at high photon energies (about $2.8 \mathrm{eV}$ ) and the biexciton line roughly $6 \mathrm{meV}$ below the exciton line. The fine structure of the spectra is caused by the finite size of the model which must be kept small to be numerically feasible. At $30 \mathrm{~K}$ and low densities the spectrum exhibits a weak but significant $(|P|=5 \%)$ signature of polarized biexciton emission with the sign opposite to

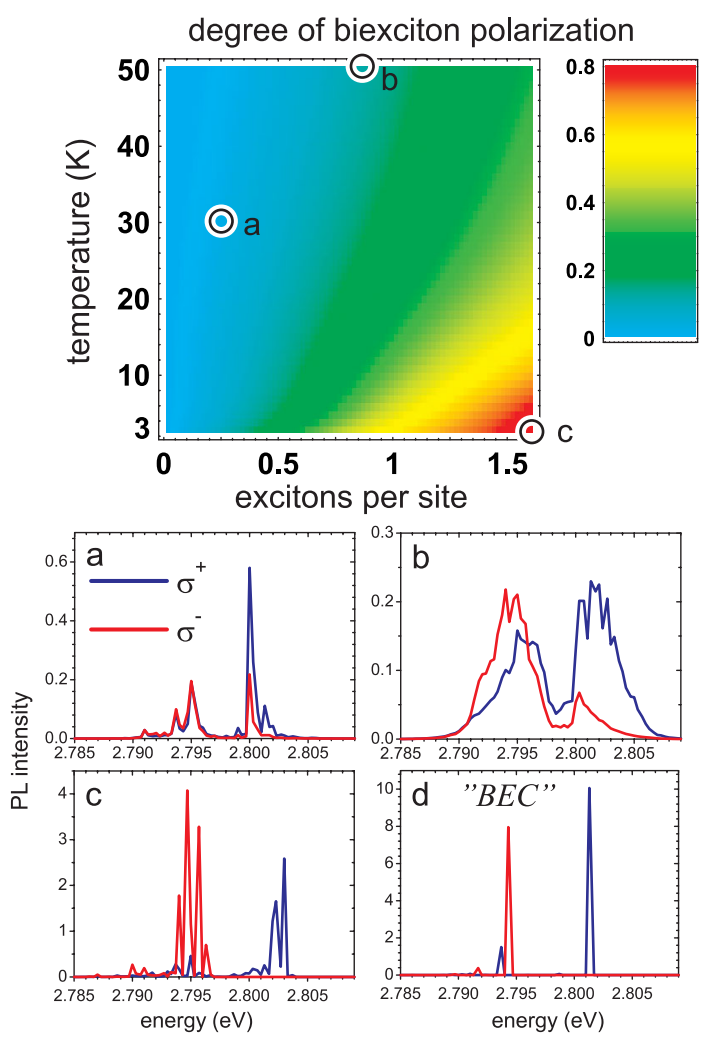

FIG. 1 (color online). (top) Degree of circular polarized biexciton luminescence $|P|$ calculated within the seven site model. (a)-(c) Calculated PL spectra for different points in the temperature-density space (see color plot above). (d) Polarized PL spectrum for $T=0 \mathrm{~K}$ corresponding to the BEC case with a total of four spin +1 and two spin -1 excitons (density of 0.86 excitons per site).

the exciton polarization [Fig. 1(a)]. Increased densities and higher temperatures lead to an expected broadening of both the exciton and biexciton line [Fig. 1(b)]. Approaching the extreme conditions of low temperatures and high densities [Fig. 1(c) and 1(d)], we find three features: (i) the emission lines narrow as predicted by Blatt, Böer, and Brandt [3], (ii) the exciton line shows a blue shift, and (iii) the degree of biexciton polarization reaches an extremum with the sign opposite to the exciton polarization. The exciton line is completely polarized because excitons with opposite spins are bound into the energetically more favorable biexcitons. We conclude from the calculated spectra that apart from narrow spectral lines also a high degree of biexciton polarization is required for the claim of a degenerate exciton gas and ultimately BEC (Kosterlitz-Thouless transition in two dimensions). Increasingly sharp exciton lines can also occur in the limit of low densities at moderate temperatures [Fig. 1(a)] and are therefore not sufficient to prove $\mathrm{BEC}$. In the case of BEC, we moreover expect a sudden and clear appearance of all described spectral features (i) to (iii).

The BPE found in the numerical treatment above will in the following purely analytical discussion be traced back to the bosonic behavior of excitons. The BPE can be com- 
pared to stimulated emission of photons where the excitons take on the role of the photons enhancing the optical decay of biexcitons. The influence of the fermionic constituents of the exciton, electron and hole, will be clarified. The temporal derivative of the dipole operator for photons with angular momentum $s$ (Heisenberg picture, no approximations) $[15,18]$

$$
\frac{\hbar}{i} \frac{\partial}{\partial t} D_{s}=-\left(E_{0} D_{s}+V_{B} d \sum_{j} X_{j,-s}^{\dagger} X_{j, 1} X_{j,-1}\right)
$$

exhibits one term related to the decay of an exciton and a second term $D_{s}^{(B)}=d \sum_{j} X_{j,-s}^{\dagger} X_{j, 1} X_{j,-1}$ where a pair of excitons with opposite spins on the same site is destroyed and an exciton with spin $-s$ is created. This process is known to be the dominant process in biexciton recombination [11]. In thermal equilibrium $D_{s}^{(B)}$ acts as a stochastic driving term of $D_{s}$, which in turn governs the PL. The intensity of the biexciton PL $I_{s}^{(B)}$ is therefore proportional to the variance (the modulus square) of $D_{s}^{(B)}$. After introducing the biexciton operator in momentum space $b_{k, s}=$ $N^{-1 / 2} \sum_{j} X_{j, 1} X_{j,-1} \exp [2 \pi i(k j / N)]$, we obtain

$$
\begin{aligned}
I_{s}^{(B)} & \propto\left\langle D_{s}^{(B)^{\dagger}} D_{s}^{(B)}\right\rangle=|d|^{2} \sum_{k, k^{\prime}}\left\langle b_{k}^{\dagger} x_{k,-s} x_{k^{\prime},-s}^{\dagger} b_{k^{\prime}}\right\rangle \\
& \approx|d|^{2} \sum_{k}\left\langle b_{k}^{\dagger} x_{k,-s} x_{k,-s}^{\dagger} b_{k}\right\rangle \\
& =|d|^{2} \sum_{k}\left\langle b_{k}^{\dagger}\left(1+x_{k,-s}^{\dagger} x_{k,-s}-\frac{2 n_{s}}{N}\right) b_{k}\right\rangle \\
& \approx|d|^{2} \sum_{k}\left\langle b_{k}^{\dagger} b_{k}\right\rangle\left(1+\left\langle x_{k,-s}^{\dagger} x_{k,-s}\right\rangle-\frac{2\left\langle n_{s}\right\rangle}{N}\right) .
\end{aligned}
$$

The off-diagonal $k$-dependent contributions disappear in the second line if an incoherent momentum distribution of excitons and biexcitons is assumed. After employing the Bose-like commutation relation in the third line (no approximation, see [15]), we approximate the four operator expectation values by two operator expectation values. We find that the biexciton luminescence intensity depends not only on the occupation of biexcitons $\left\langle b_{k}^{\dagger} b_{k}\right\rangle$ as naively expected. The intensity is in fact enhanced by an additional factor $\left(1+\left\langle x_{k,-s}^{\dagger} x_{k,-s}\right\rangle-\frac{2\left\langle n_{s}\right\rangle}{N}\right)$ which increases with the occupation $\left\langle x_{k,-s}^{\dagger} x_{k,-s}\right\rangle$ of exciton states. The excitons involved in the enhancement possess the opposite angular momentum as the photons emitted in the biexciton decay. The first two terms of the enhancement factor originate from the bosonic properties of the excitons and correspond to the well-known stimulated-scattering factor " $1+n$ " that appears in stimulated-scattering events in quasi-ideal bosonic systems like lasers or atomic BECs. The bosonic enhancement is counteracted by an additional third term $-2\left\langle n_{s}\right\rangle / N$ which reminds us that an exciton is made of two fermions which are subject to Pauli blocking at high densites [19]. The biexcitonic emissions $I_{+1}^{(B)}$ and $I_{-1}^{(B)}$ are equally strong only if the degree of exciton spin polariza- tion is zero. If the biexcitons coexist with a majority of spin up $(+1)$ excitons, the spin dependent enhancement factor breaks the symmetry of the biexciton decay channels, resulting in negatively polarized biexciton emission explaining the appearance of the BPE in the calculated spectra. The polarization degree of the BPE $P=\left(I_{1}^{(B)}-\right.$ $\left.I_{-1}^{(B)}\right) /\left(I_{1}^{(B)}+I_{-1}^{(B)}\right)$ is therefore a natural measure for the deviation from classical towards bosonic behavior of the exciton gas and a key figure in experiments for verifying bosonic behavior of excitons. The biexciton polarization has in a finite model of $N$ sites an upper limit of $|P|<[(N+$ $1)-1] /[(N+1)+1]$. Complete polarization is expected in large systems, where a macroscopic occupation of the $k=$ 0 exciton state appears at low temperatures. Polarized PL of the biexciton will also occur in two- and threedimensional systems in analogy to the treatment above.

Last we present experimental evidence for the BPE in a single ZnSe QW using time and polarization-resolved PL measurements. Polarization artifacts are excluded by control experiments: (i) changing the sign of the circularly polarized laser pump pulse led to a fully symmetric change in the observed polarized PL, (ii) temporal spin oscillations in an external magnetic field caused oscillations in both the exciton PL polarization and the biexciton PL polarization, (iii) linear polarized laser excitation led to $0 \pm 0.5 \%$ circular polarization of the detected PL. The following list of possible effects in the sample reduce the BPE but cannot fake the BPE: (i) reabsorption of light emitted at the (a)

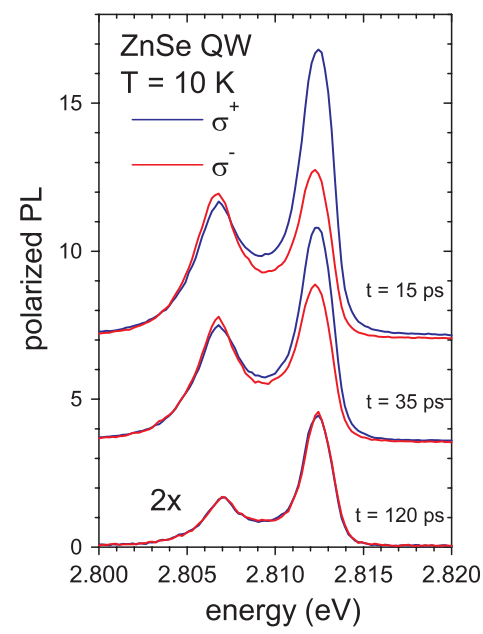

(b)

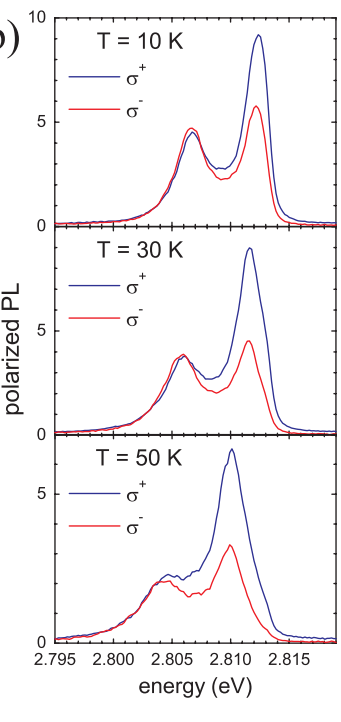

FIG. 2 (color online). (a) Transient, polarization-resolved PL spectra of a $10 \mathrm{~nm} \mathrm{ZnSe}$ quantum well after resonant pulsed laser excitation. The early spectra exhibit negatively polarized biexciton emission consistently evidencing the biexciton polarization effect. After $120 \mathrm{ps}$, exciton spin polarization has disappeared (with equal $\sigma^{+}$- and $\sigma^{-}$-PL intensity). (b) Polarization-resolved PL spectra for increasing lattice temperatures. The absolute degree of biexciton polarization decreases from $5 \%$ at 10 and $30 \mathrm{~K}$ to $0 \%$ at $50 \mathrm{~K}$. 
biexciton line, since the bosonically enhanced decay channel shows stronger reabsorption due to the presence of majority excitons there, (ii) a tiny effect opposite to the BPE, found when we simulated the Purcell effect induced by a polarization dependent refractive index due to bleaching, (iii) biexciton PL superposed by the low energy tail of the polarized exciton PL.

Figure 2 shows PL spectra taken from a $10 \mathrm{~nm}$ thick ZnSe QW embedded in $500 \mathrm{~nm} \mathrm{ZnS}_{0.07} \mathrm{Se}_{0.93}$ barriers grown by molecular beam epitaxy on GaAs substrate. Similar data are obtained for an $8 \mathrm{~nm}$ ZnSe multiple QW and a $9.9 \mathrm{~nm}$ thick GaAs single QW. The $\mathrm{ZnSe}$ single QW exhibits large exciton binding energies $(>16 \mathrm{meV})$ and a large heavy-hole light-hole splitting $(>25 \mathrm{meV})$ which makes the system an almost perfect two band semiconductor. A fundamental absorption linewidth of $0.8 \mathrm{meV}$ and a homogeneous linewidth of $0.65 \mathrm{meV}$ measured by four wave mixing spectroscopy demonstrate that disorder is negligible [20]. Excitons with spin +1 are created in the sample by circularly polarized ps pulses from a frequency doubled $80 \mathrm{MHz}$ Ti:sapphire laser. The sample is kept at temperatures between 10 and $50 \mathrm{~K}$. The laser energy is tuned to the high energy tail of the heavy-hole resonance to resonantly create a cold exciton gas and reduce stray light at the heavy-hole PL line. The left and right circularly polarized PL components are detected by a spectrometer and a streak camera system with a spectral and temporal resolution of $2 \mathrm{meV}$ and $6 \mathrm{ps}$, respectively. The setup reaches a polarization sensitivity of $0.5 \%$ using an electrically switchable liquid crystal retarder before the polarizer and streak camera.

Spin -1 excitons appear due to rapid spin relaxation already a few ps after excitation, giving rise to the formation of biexcitons from pairs of excitons with opposite spins. Excitons and biexcitons then coexist in a dynamic quasiequilibrium until all carriers decay by optical recombination on a time scale of typically less than $0.5 \mathrm{~ns}$. Figure 2(a) displays polarization-resolved PL spectra 15, 35 , and $120 \mathrm{ps}$ after laser excitation. The exciton density of about $10^{11} \mathrm{~cm}^{-2}$ is well below the Mott density. At that density thermal equilibrium is always established, allowing for comparison of experiment with theory (see above). The exciton PL exhibits at early times a high degree of circular polarization since the majority of excitons have spin +1 . Most importantly, the biexciton emission exhibits a significant degree of polarization of about $-5 \%$ at $t=$ 15 ps that has the opposite sign compared to the exciton PL polarization in agreement with the predicted BPE. A higher degree of biexciton polarization would require lower carrier temperatures. Those cannot be obtained in our experiment, because the binding energy that must be dissipated in the biexciton formation process heats the distribution of excitons above lattice temperature. We find completely unpolarized spectra $120 \mathrm{ps}$ after laser excitation when the carrier spin has completely relaxed. We also observe the disappearance of polarized biexciton PL at elevated temperatures and fixed densities which is predicted for the BPE by our numerics. Figure 2(b) shows the polarization-resolved PL spectra taken 15 ps after laser excitation at 10, 30, and $50 \mathrm{~K}$ sample temperature. The spectra at 10 and $30 \mathrm{~K}$ clearly exhibit the signature of the BPE. The biexciton polarization at $50 \mathrm{~K}$ seems to have reversed the sign, but a line fit which filters out the contribution of the exciton line reveals that the true biexciton polarization is zero within the error bars. A prospective experimental proof of BEC will require lower carrier temperatures that may be obtained by simultaneous creation of excitons and biexcitons via spectrally shaped laser pulses which avoids heating of the exciton gas via the biexciton formation process.

In conclusion, we presented the biexciton polarization effect as a novel signature for bosonic behavior. Our findings contribute to the long-standing question of stimulated exciton scattering and BEC in semiconductors. Photoluminescence experiments verify the existence of the biexciton polarization effect both in $\mathrm{ZnSe}$ and GaAs quantum wells.

We gratefully acknowledge the financial support by the DFG and the BMBF and helpful discussions with E. Jeckelmann and R. Zimmermann. S.P. thanks the Friedrich Ebert foundation for financial support.

*Present address: Fakultät für Physik und Astronomie, Ruhr-Universität Bochum, D-44780 Bochum, Germany.

[1] M. H. Anderson et al., Science 269, 198 (1995).

[2] M. R. Andrews et al., Science 275, 637 (1997).

[3] J. M. Blatt, K. W. Böer, and W. Brandt, Phys. Rev. 126, 1691 (1962).

[4] G. Dasbach et al., Phys. Rev. B 70, 045206 (2004).

[5] J. Fernández-Rossier, C. Tejedor, and R. Merlin, Solid State Commun. 108, 473 (1998).

[6] D. Snoke, Science 298, 1368 (2002).

[7] R. Rapaport and G. Chen, J. Phys. Condens. Matter 19, 295207 (2007).

[8] D. Snoke, Nature Phys. 4, 674 (2008).

[9] J. Kasprzak et al., Nature (London) 443, 409 (2006).

[10] H. T. Cao, T. D. Doan, D. B. Tran Thoai, and H. Haug, Phys. Rev. B 77, 075320 (2008).

[11] J. C. Kim, D. R. Wake, and J. P. Wolfe, Phys. Rev. B 50, 15099 (1994).

[12] M. Kira, F. Jahnke, W. Hoyer, and S. W. Koch, Prog. Quantum Electron. 23, 189 (1999).

[13] K. Hannewald, S. Glutsch, and F. Bechstedt, Phys. Rev. B 62, 4519 (2000).

[14] V. M. Agranovich and S. Mukamel, Phys. Lett. A 147, 155 (1990).

[15] See EPAPS Document No. E-PRLTAO-103-036938 for supplementary material containing detailed derivations. For more information on EPAPS, see http://www.aip.org/ pubservs/epaps.html.

[16] R. A. Kaindl, D. Hägele, M. A. Carnahan, and D. S. Chemla, Phys. Rev. B 79, 045320 (2009).

[17] H. Kalt et al., Phys. Status Solidi B 206, 103 (1998).

[18] W. Schäfer et al., Phys. Rev. B 53, 16429 (1996).

[19] H. Haug and S. Schmitt-Rink, Prog. Quantum Electron. 9, 3 (1984).

[20] T. Voß, Ph.D. thesis, Universität Bremen, 2004. 